\title{
Assessment of Sliding Mode Observer in Sensorless Control of Switched Reluctance Motors
}

\author{
N. K. Dankadai, M. A. Elgendy, S. P. Mcdonald, D. J. Atkinson, G. Atkinson \\ School of Engineering, Newcastle University, Merz Court, Newcastle Upon Tyne, NE1 7RU, UK \\ n.k.dankadai2@newcastle.ac.uk
}

\begin{abstract}
Several studies have proved that sensorless control methods can improve the overall robustness and costs of a drive while maintaining the desired performance. However, the general approach of these sensorless strategies involves flux estimation which is significantly affected by the uncertainties associated with the machine's terminal measurement errors and noise. In this paper, a simple robust sensorless control of Switched Reluctance Machine (SRM) is described. The inherent robustness to parameter variations and measurement noise coupled with the high stability and simple computation of a Sliding Mode Observer (SMO) is utilized to eliminate the errors often involved in a fluxlinkage based position estimation for the SRM. The proposed method is tested under different operating conditions. Results obtained show that it is reliable and less susceptible to errors and noise commonly found in sensorless control of SRM. The method was also found to handle the model uncertainties associated with the approximated model used for the estimation but with reduced performance at low speed.
\end{abstract}

\section{INTRODUCTION}

The SRM has gained more interest in applications for electric vehicles, the more-electric aircraft, industry and white goods over the last decade. This is mainly because of its lowcost, fault-tolerance, simple structure and wide speed range of operation [1]-[4]. The SRM controller needs accurate and rapid information of its rotor position to work effectively due to its principle of operation. A separate position sensor is conventionally used to detect rotor position but adds size, weight and cost to the drive. Furthermore, position sensors tend to reduce the reliability of the drive as they add additional physical connections and can be vulnerable to environmental factors. Therefore, an encoder-less scheme can be used to estimate SRM's rotor position via the machine's terminal current and voltage measurements [5][6]. Nevertheless, these measured feedback signals are often affected by measurement noise and errors within the acquisition chain. Hence, the efficacy of the estimation method depends on the robustness of the estimation technique to these measurement uncertainties.

Generally, estimation of rotor position in the SRM drive is based on the relationship between the machine's phase current, phase flux-linkage and rotor position. It is impractical to directly measure the flux-linkage, thus, it is often estimated by integrating the voltage across an excited phase which requires measurement of phase current and phase voltage. However, the accuracy of the estimation is affected by several error components summarized in Table 1.

Various studies have been carried out to reduce errors in flux estimation for sensor-less control of an SRM. Yan et'al proposed a circuit-based flux-linkage measurement method with an automated winding resistance correction method to eliminate errors in sensorless control of SRM. This method requires additional circuit components [7]. Other flux-linkage estimation strategies with error reduction that require no additional hardware are as follows: In [8] a DSP-based automated error-reduction method in flux-linkage measurement for SRM has been proposed, it includes online offset correction and phase resistance estimation in a virtual instrumentation form. Wenkai et'al designed an integral flux error correction technique to reduce phase resistance error cause by temperature variation. A feedback regulator is employed to correct the resistance when the phase current is zero [9]. Peng et al reported the use of a third order phaselocked loop to reduce the effect of SRM terminal measurement noise and numerical measurement residual error on a numerical method of flux estimation [10]. The implementations of artificial intelligence based flux-linkage prediction methods to eliminate the impact of parameter variation, measurement error in SRM flux estimation were also reported. These methods take advantage that the error accumulation in SRM flux estimation resets at the end of each electrical cycle [11]-[13].In summary, methods employed for position estimation that are based on flux-linkage models often require additional function to correct the flux calculation-error hence increase real-time computation.

In this paper, an inductance model based Sliding Mode Observer (SMO) is employed to estimate SRM position without the requirement of additional functions for fluxlinkage calculation and machine-terminal measurement error corrections. It uses only operating signals, so no additional sensors or external circuitry are needed. Its computational simplicity and robustness make it a superior choice for sensorless control of SRM drives.

TABLE I. SOURCES OF FEEDBACK ERROR IN SRMS

\begin{tabular}{|l|l|}
\hline Measurement Errors & $\begin{array}{l}\text { - Sensor offset error } \\
\text { - Sensor scaling error }\end{array}$ \\
\hline Measurement Noise & $\begin{array}{l}\text { - Capacitive coupling of measuring circuit } \\
\text { - Electromagnetic-interference from the } \\
\text { motor, Power circuit and other electronic } \\
\text { devices within its vicinity. }\end{array}$ \\
& $\begin{array}{l}\text { - Integration offset error } \\
\text { - Time and amplitude quantization error } \\
\text { Flux-linkage }\end{array}$ \\
$\begin{array}{l}\text { Calculation Error } \\
\text { - Variation of phase resistance due to } \\
\end{array}$ & \\
\hline
\end{tabular}




\section{INDUCTANCE-BASED MODEL OF SRM}

A model of the SRM is needed to be derived to define a sliding mode observer that can be used to estimate its rotor position. The dynamics of an SRM can be modelled from the SRM drive system differential equations which include the electromagnetic equations, the electromechanical equations, and the mechanical equations follows:

The voltage equation (electromagnetic equations) is a nonlinear differential equation (1):

$$
V_{j}=i_{j} \cdot R+L_{j}(\theta, i) \frac{d i_{j}}{d t}+i_{j} \frac{\partial L_{j}(\theta, i)}{\partial \theta} \cdot \omega+i_{j} \frac{\partial L_{j}(\theta, i)}{\partial i_{j}} \frac{d i_{j}}{d t} .
$$

with $\mathrm{j}=1 \ldots \mathrm{N}$, where $\mathrm{N}$ is the number of phases, $V$ is the applied phase voltage, $R$ is the winding resistance per phase, $\omega$ is angular velocity and $L$ is the phase inductance at a phase current $i$ and rotor position $\theta$, which is defined as " $L=\Psi(\theta, i) / i$ " and $\Psi$ is the phase flux-linkage. Therefore, the rate of change of current of a given SRM can be calculated from (1) as depicted in (2):

$$
\frac{d i_{j}}{d t}=\left(V_{j}-i_{j} \frac{\partial L_{j}(\theta, i)}{\partial \theta} \cdot \omega\right) /\left(L_{j}(\theta, i)+i_{j} \frac{\partial L_{j(\theta, i)}}{\partial i_{j}}\right)=\frac{X(\theta, i, \omega)}{Y(\theta, i)}
$$

The torque-speed equation (electromechanical equations) can be expressed as:

$$
J \frac{d \omega}{d t}=\sum_{j=1}^{N} T_{e_{j}}-T_{L}
$$

where $T_{e_{j}}$ and $T_{L}$ are phase torque and load torque, respectively. The resultant torque can be obtained by the summing up the individual phase torques as expressed below:

$$
T_{e}=\sum_{j=1}^{N} \frac{\partial W_{c_{j}}}{\partial \theta}=\quad \sum_{j=1}^{N} \frac{\partial \int L_{j}(\theta, i) i_{j} d i}{\partial \theta}
$$

Therefore, the angular acceleration of the motor can be expressed as:

$$
\frac{d \omega}{d t}=\frac{\sum_{j=1}^{N} T_{e_{j}}-T_{L}}{J}=Z(\theta, i)
$$

The mechanical equation can be expressed as:

$$
\frac{d \theta}{d t}=\omega
$$

Based on equations (2) to (6), the overall dynamic response of the SRM drive can be calculated.

\section{DEFINITION OF SLIDING MODE OBSERVER}

The inductance of the model used in defining a SMO for SRMs can be derived from finite terms of Fourier series with the coefficients of the series representing the non-linear change of inductance with the phase current [14]. These coefficients can be generated in the form of polynomial functions from the aligned-position inductance, unaligned-position inductance and middle-position inductance.

Therefore, the individual phase inductance can be expressed as in (7)

$$
L(\theta, i)=\sum_{n=0}^{\infty} A_{n}(i) \cdot \cos \left(n N_{r} \theta+\varphi_{n}\right)
$$

where $N_{r}$ is the number of rotor poles, $A_{n}$ are coefficients of the series. Their values are generally obtained from curve fitting and stored in a look-up table.

At this point, a second order sliding mode observer for rotor position and speed estimation of SRM can be defined as in (8) and (9), respectively, according to the system differential equations derived in the previous section:

$$
\begin{gathered}
\dot{\bar{\theta}}=\bar{\omega}+K_{\theta} \operatorname{sgn}\left(E_{i}\right) \\
\dot{\bar{\omega}}=Z(\bar{\theta}, \bar{\imath})+K_{\omega} \operatorname{sgn}\left(E_{i}\right)
\end{gathered}
$$

where $\bar{\omega}, \bar{\theta}, \bar{l}$, are the estimations of $\omega, \theta, \mathrm{i}$, and $Z(\bar{\theta}, \bar{\imath})$ is the estimated angular acceleration and the function $\operatorname{sgn}(-)$ returns the sign of its operand.

The variable $E_{i}$ is an error function based on the difference between the measured and estimated Phase current which is used to stabilize the error dynamics of the estimation. In this study, the following error function is used:

$$
\left.E_{i}=\sum_{j=1}^{N} \overline{(l}_{j}-i_{j}\right)
$$

To describe the observer error dynamics, the following estimation errors are defined:

$$
\begin{aligned}
& E_{\theta}=\theta-\bar{\theta} \\
& E_{\omega}=\omega-\bar{\omega}
\end{aligned}
$$

Differentiating (11) and (12) yields (13) and (14) respectively:

$$
\begin{aligned}
& \dot{E}_{\theta}=\dot{\theta}-\dot{\bar{\theta}} \\
& \dot{E}_{\omega}=\dot{\omega}-\dot{\bar{\omega}}
\end{aligned}
$$

By substituting (8) in and (13) in (9) and (14) respectively (15) and (16):

$$
\begin{gathered}
\dot{E}_{\theta}=\dot{\theta}-\bar{\omega}+K_{\theta} \operatorname{sgn}\left(E_{i}\right) \\
\dot{E}_{\omega}=\dot{\omega}-Z(\bar{\theta}, \bar{\imath})+K_{\omega} \operatorname{sgn}\left(E_{i}\right)
\end{gathered}
$$

Which can also be represented as (17) and (18) respectively:

$$
\begin{gathered}
\dot{E}_{\theta}=E_{\omega}+K_{\theta} \operatorname{sgn}\left(E_{i}\right) \\
\dot{E}_{\omega}=Z(\theta, i)-\left[Z(\bar{\theta}, \bar{\imath})+K_{\omega} \operatorname{sgn}\left(E_{i}\right)\right]
\end{gathered}
$$

Since the gain $K_{\omega}$ is often selected to be large enough, then $Z(\theta, i)-Z(\bar{\theta}, \bar{\imath})$ in (12) can be approximated as in (19).

$$
\dot{E}_{\omega}=-K_{\omega} \operatorname{sgn}\left(E_{i}\right)
$$


From (13), it can be found that if the error function $E_{i}$ is selected to have the same sign as $E_{\theta}$, and the observer gain $K_{\theta}$ is selected to have:

$$
K_{\theta}>\left|E_{\omega}\right|
$$

Then $E_{\theta}$ will always have a different sign from $\dot{E}_{\theta}$ and the sliding surface $E_{\theta}=0$ will be reached in a finite time, then the position estimated rotor position $\bar{\theta}$ will be equal to the actual rotor position $\theta$, hence, the estimated angular speed $\bar{\omega}$ converges exponentially to the actual speed $\omega$.

\section{SIMULATION RESUlTS AND DISCUSSION}

The sliding mode observer defined above has been developed in MATLAB/Simulink. The actual motor model and the estimation model were created using the measurement data from the motor and Fourier series approximated model respectively. With a practical system, the voltage and current measurements are often corrupted by noise. To test the developed observer under the effect of measurement error and noise which include sensor offset error, sensor scaling errors, and measurement noise due to capacitive coupling of measuring circuit, electromagnetic-interference from the motor, power circuit and other electronic devices within its vicinity were added to the actual motor terminals. Fig. 1 depicts the hysteresis current control used for the analysis.

However, it is difficult to quantify each error component in the instantaneous measurements of the machine's feedback signal because their causal factors are system dependent and time-varying. Therefore, for analysis, an error amplitude can be assumed to represent the total measured current and voltage error.

Ideally, the effect of both current and voltage position measurement together with the estimation-model uncertainties should be considered when analysing the error involved in the position and speed estimation.

The effects of each phase voltage and phase current error in the rotor position and speed estimation are represented by a resultant error of $10 \%$ in the phase voltage measurement and $10 \%$ in phase current measurement. A white noise with a $10 \%$ amplitude and $20 \mathrm{kHz}$ sampling time is used in the simulation to represent the errors.

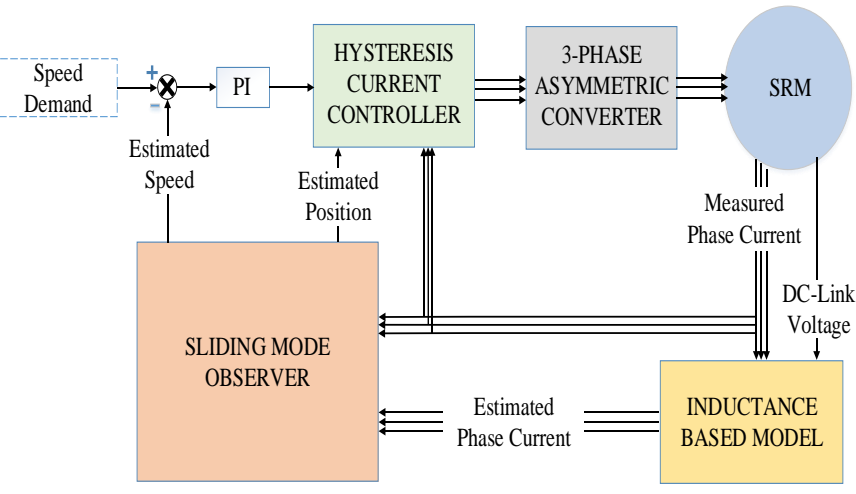

Fig. 1. Sliding Mode Observer based current control
Figs. 2 and 3 depict the phase current waveforms and the phase voltage waveforms respectively, both with and without measurement error at $500 \mathrm{rpm}$. This is within the middle of the speed range for the SRM under investigation. The effect of the error on the measured phase voltage and phase current signals can be observed to cause a significant change. This change can be employed to analyze the accuracy of the SMO under the efferent of measurement noise and error.

Furthermore, SRMs are highly nonlinear machines. It is difficult to obtain their linear dynamic models in form of a transfer function which is used for controller design and analysis. Nevertheless, a Fourier series non-linear inductance model as expressed in the SRM model section above can be employed. The model is used to calculate the instantaneous phase current of the SRM required for the SMO based estimation which is also expressed in the SMO definition section above. Hence, the model is generally not ideal due to the inherent high non-linearity and tine varying properties of SRMs.

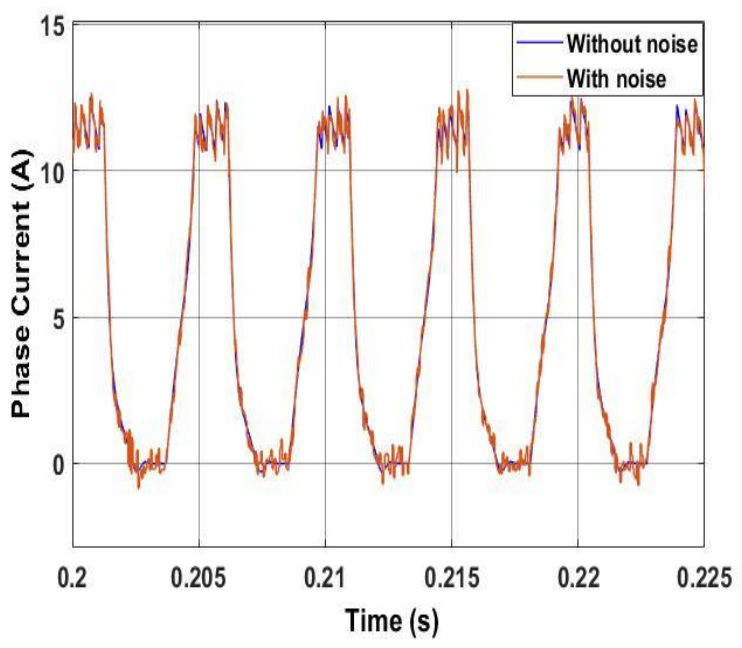

(a)

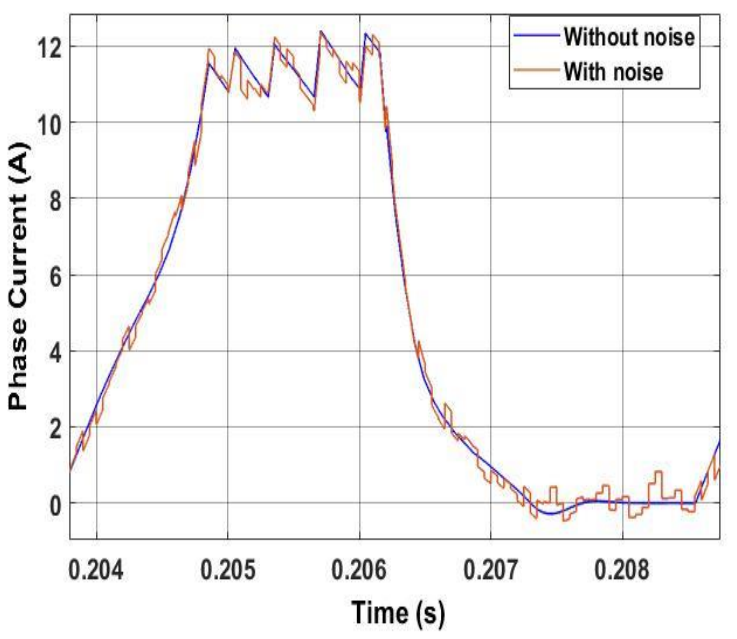

(b)

Fig. 2. SRM phase current (a) with and without measurement error comparison (b) expanded comparison. 


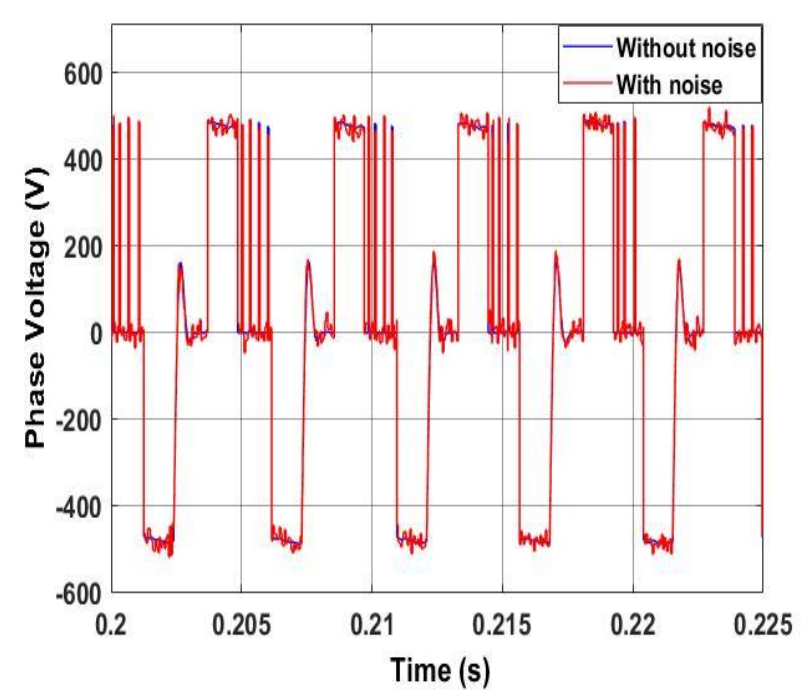

(a)

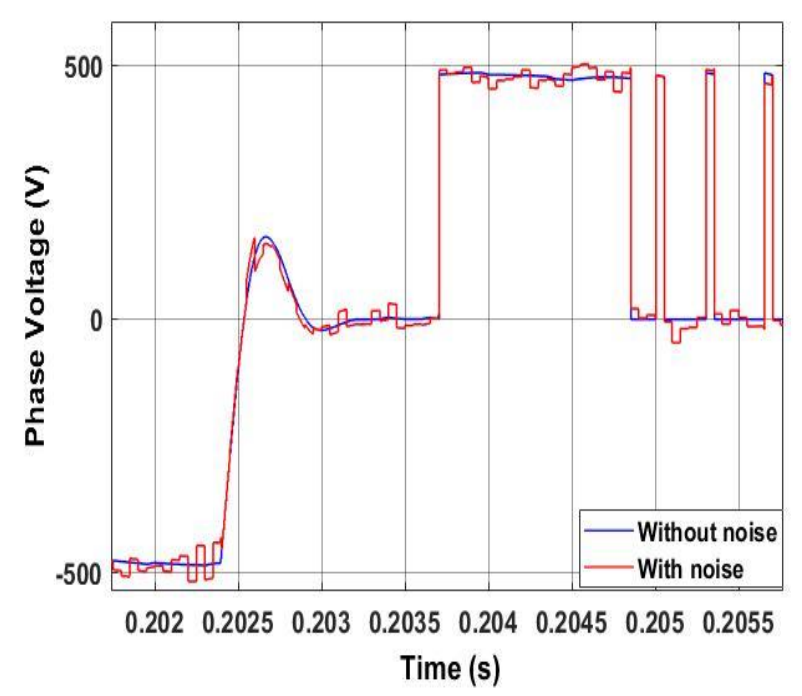

(b)

Fig. 3. SRM phase voltage (a) with and without measurement error comparison (b) expanded comparison.

A good estimation methods should have a reasonable robustness to uncertainties. Therefore, small deviation of the feedback signals from its true value due to the aforementioned measurement error and noise, together with the model uncertainty should only decrease its accuracy by a small and reasonable amount.

Fig 4 (a) shows the comparison of the actual and estimated phase current at $500 \mathrm{rpm}$. The difference between these phase currents is used to calculate the sliding surface of the SMO. It can be easily seen that the phase currents are not exactly equal as a result of the measurement error and model uncertainties. The error across the SRM range of speed is depicted in Fig 4 (b) which tends to be higher at near zero speed and the phase commutation region.

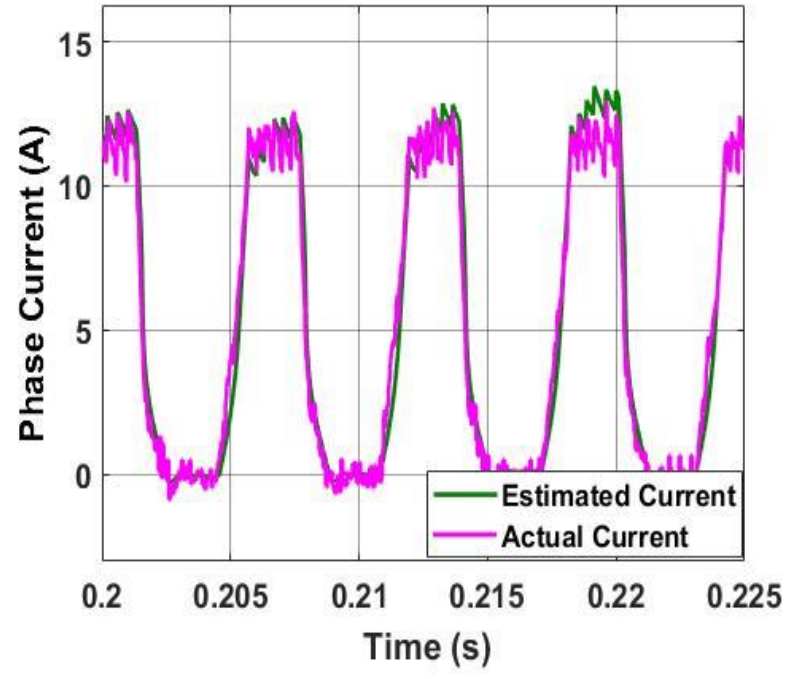

(a)

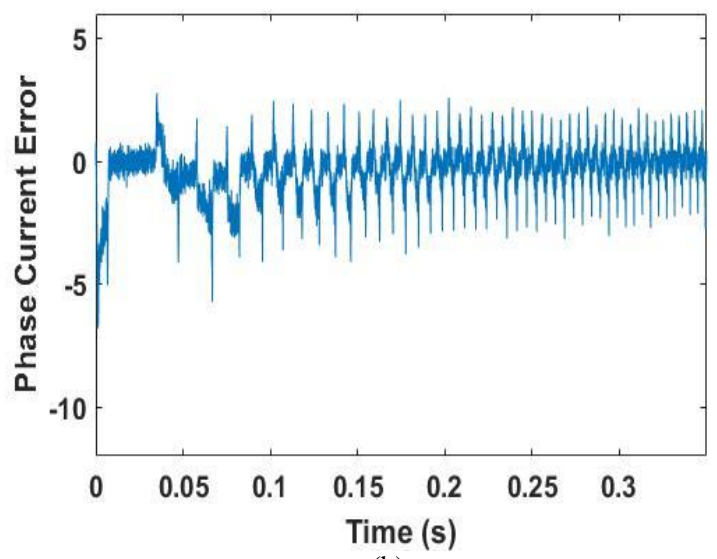

(b)

Fig. 4. Comparison of actual and estimated phase current. (a) phase currents at medium speed range (b) current error across speed range of the SRM

In Fig 5, the waveform of the comparisons between actual and estimated rotor position and speed of the SRM running at a steady speed of $500 \mathrm{rpm}$ is shown. It can be observed that the results show an acceptable accuracy in estimation of both rotor position and speed within the medium speed range.

From Fig 7, a very low-speed results of the position estimation is shown. It can be seen that the observer produced a large error at near zero speed. This is because regular operating electrical signals are not available within this region. Hence, a larger error from the estimation-model calculation is produced which affects the rate of convergence of the SMO. In addition, the noise effect is also more significant when compared to the available measured feedback signals. So SMO often fails at near zero speed or very high speed. Therefore, a 
different technique for sensorless start-up and low-speed is required for proper operation of the SRM.

Table II summarized the performance of the proposed SMO at several speeds. It shows the absolute values of the average errors. The SMO depicted a good performance in spite the model uncertainties and the errors in feedback signals across a wide range of speed but it shows poor performance in the estimation of rotor position speed at standstill and near zero speeds. A signal injection method can be implemented in this region. The particular phase to be energized can be determined by injecting equal amount of voltage to each phase and comparing their generated maximum current. Nevertheless, the accuracy of the SMO started to decrease above the base speed of the SRM as can be seen from the average error at 900 rpm.

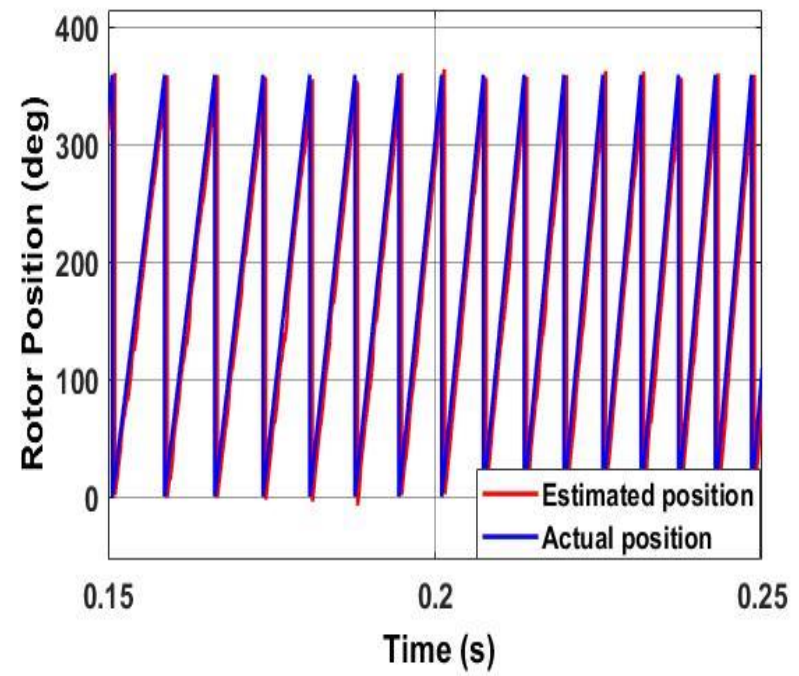

(a)

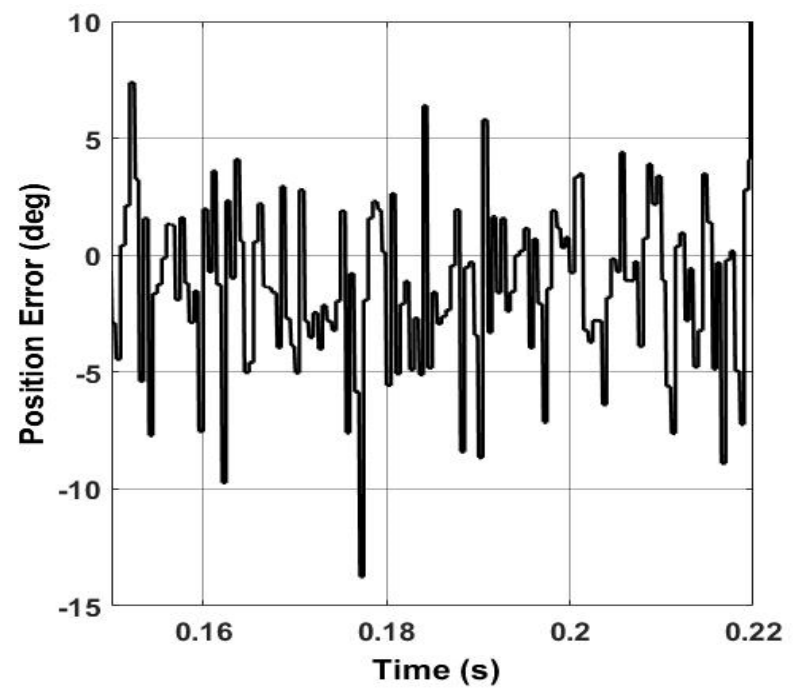

(b)

Fig. 5. Actual and SMO estimation at 500rpm (a) rotor position (b) angular speed

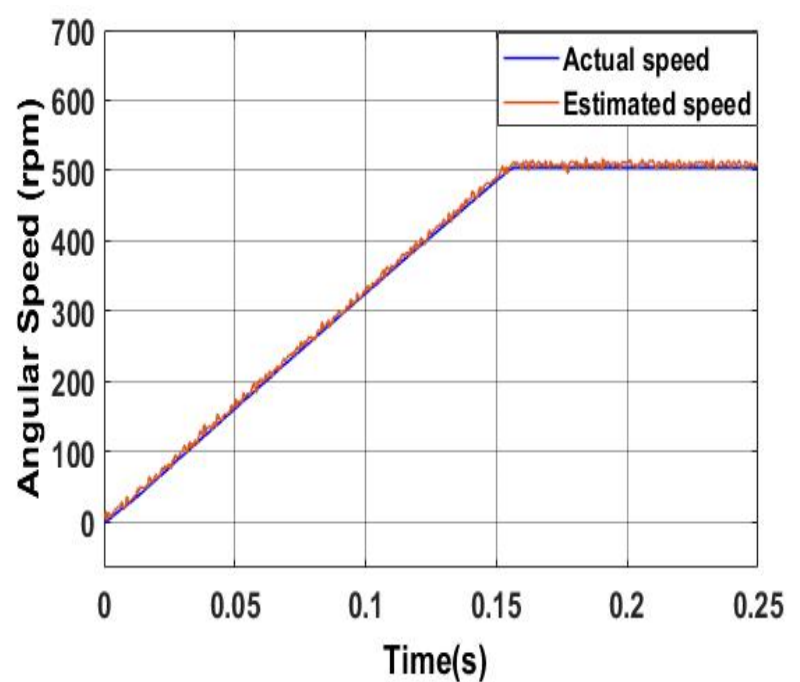

(a)

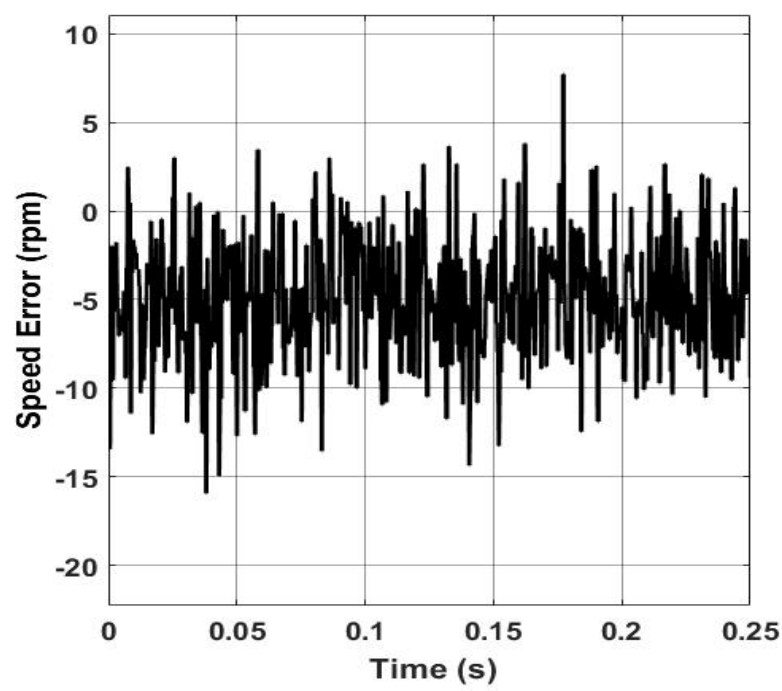

(b)

Fig. 6. Actual and SMO estimation at 500rpm (a) rotor position (b) angular speed

TABLE II

AVERAGE POSITION AND SPEED ESTIMATION ERROR USING SMO

\begin{tabular}{|c|c|c|}
\hline Reference Speed & $\begin{array}{c}\text { Position average } \\
\text { error (deg). }\end{array}$ & $\begin{array}{c}\text { Speed average } \\
\text { error (rpm) }\end{array}$ \\
\hline $\begin{array}{c}10 \mathrm{rpm} \\
\text { (Chopping mode) }\end{array}$ & 241.85 & 7.73 \\
\hline $\begin{array}{c}300 \mathrm{rpm} \\
\text { (Chopping mode) }\end{array}$ & 0.62 & 4.54 \\
\hline $\begin{array}{c}600 \mathrm{rpm} \\
\text { (Chopping mode) }\end{array}$ & 0.83 & 4.59 \\
\hline $\begin{array}{c}900 \mathrm{rpm} \\
\text { (Single pulse mode) }\end{array}$ & 1.46 & 5.09 \\
\hline
\end{tabular}




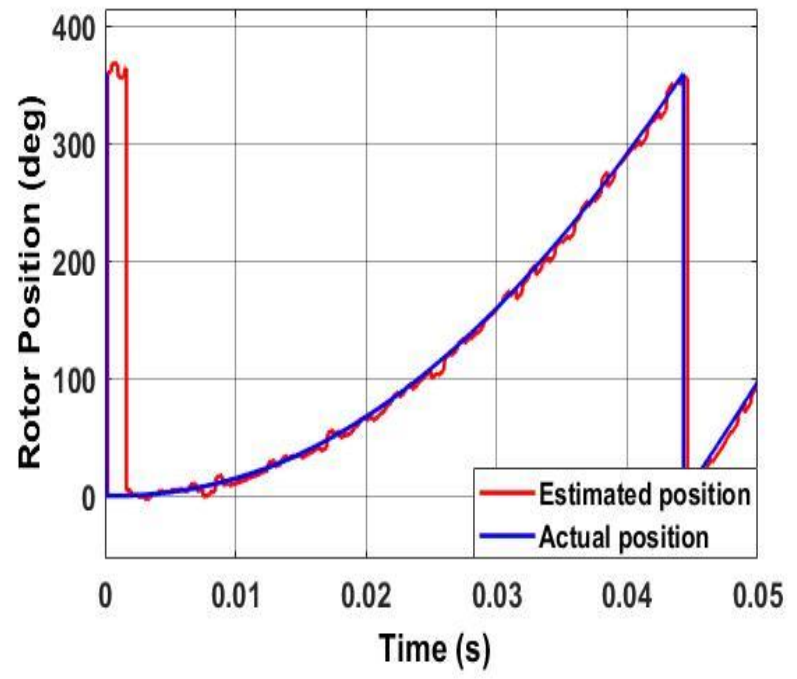

(a)

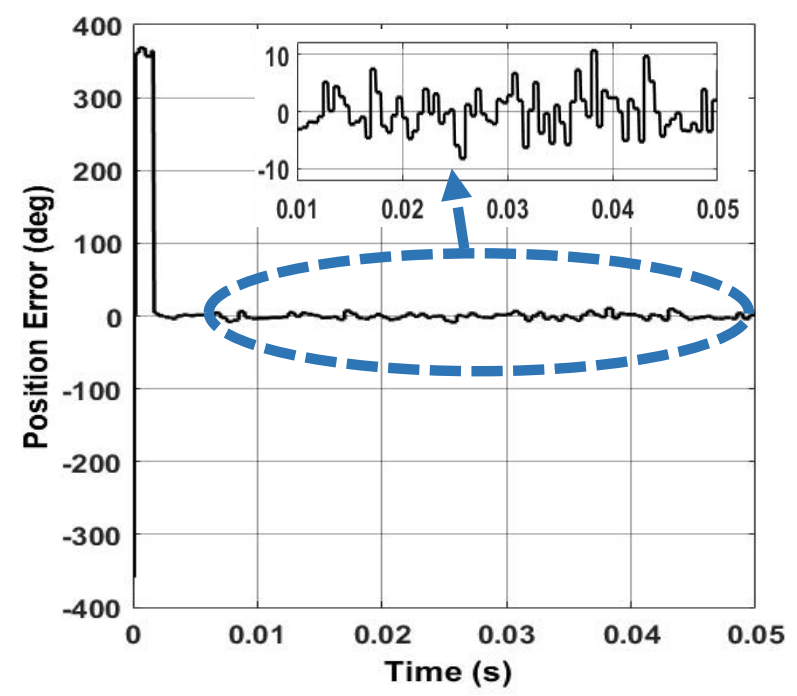

(b)

Fig. 7. Actual and SMO estimation at low speed (a) rotor position (b) Position error

\section{CONCLUSION}

In this paper, the performance of a rotor position and speed estimation method for an SRM, based on a SMO is explained and analyzed across a wide range of speed. The SMO provides a superior method to implement sensorless control of SRM. This is due to its inherent robustness to measurement errors and parameter uncertainties coupled with its computational simplicity and high stability. The observer uses measured phase voltage and phase current to perform the estimation via an inductance model of the SRM. A MATLAB/Simulink simulation model of the sensorless control was developed. The simulation results depicted that the estimation method can accurately detect the rotor position and speed of SRM under errors commonly associated with motor drives control caused by the machine's terminal measurement errors and noise, and model uncertainties. Nevertheless, it shows low performance at standstill and near zero speed range. Furthermore, its performance was found to decrease above the bases speed of the SRM but with an acceptable accuracy. A Field experiment to validate the findings is a future goal.

\section{REFERENCES}

[1] J. Borg Bartolo, M. Degano, J. Espina and C. Gerada, "Design and Initial Testing of a High-Speed 45-kW Switched Reluctance Drive for Aerospace Application," in IEEE Transactions on Industrial Electronics, vol. 64, no. 2, pp. 988-997, Feb. 2017.

[2] I. Boldea, L. N. Tutelea, L. Parsa and D. Dorrell, "Automotive Electric Propulsion Systems With Reduced or No Permanent Magnets: An Overview," in IEEE Transactions on Industrial Electronics, vol. 61, no. 10, pp. 5696-5711, Oct. 2014.

[3] K. Lu, P. O. Rasmussen, S. J. Watkins and F. Blaabjerg, "A New LowCost Hybrid Switched Reluctance Motor for Adjustable-Speed Pump Applications," in IEEE Transactions on Industry Applications, vol. 47, no. 1, pp. 314-321, Jan.-Feb. 2011.

[4] M. A. Elgendy, V. Pickert, B. Zahawi, C. Morton, A. Ayob, "Dual Voltage Supply Converter for High-Speed Doubly Salient Reluctance Motors", IEEE Transactions on Power Electronics, vol. 28, no. 2, pp. 1016-1024, Feb. 2013.

[5] B. Fahimi, G. Suresh and M. Ehsani, "Review of sensorless control methods in switched reluctance motor drives," Conference Record of the 2000 IEEE Industry Applications Conference. Thirty-Fifth IAS Annual Meeting and World Conference on Industrial Applications of Electrical Energy (Cat. No.00CH37129), Rome, Italy, 2000, pp. 1850-1857 vol.3.

[6] A. Kawamura, "Survey of position sensorless switched reluctance motor control," Proceedings of IECON'94 - 20th Annual Conference of IEEE Industrial Electronics, Bologna, Italy, 1994, pp. 1595-1598 vol.3.

[7] Y. Liang and H. Chen, "Circuit-based flux linkage measurement method with the automated resistance correction for SRM sensorless position control," in IET Electric Power Applications, vol. 12, no. 9, pp. 13961406, 112018.

[8] A. D. Cheok and Z. Wang, "DSP-Based Automated Error-Reducing Flux-Linkage-Measurement Method for Switched Reluctance Motors," in IEEE Transactions on Instrumentation and Measurement, vol. 56, no. 6, pp. 2245-2253, Dec. 2007.

[9] Wei, Q. Wang and R. Nie, "Sensorless control of double-sided linear switched reluctance motor based on simplified flux linkage method," in CES Transactions on Electrical Machines and Systems, vol. 1, no. 3, pp. 246-253, September 2017.

[10] F. Peng, J. Ye and A. Emadi, "Position sensorless control of switched reluctance motor based on numerical method," 2016 IEEE Energy Conversion Congress and Exposition (ECCE), Milwaukee, WI, 2016, pp. 1-8.

[11] R. Zhong, Y. B. Wang and Y. Z. Xu, "Position sensorless control of switched reluctance motors based on improved neural network," in IET Electric Power Applications, vol. 6, no. 2, pp. 111-121, February 2012.

[12] A. D. Cheok and N. Ertugrul, "High robustness and reliability of fuzzy logic based position estimation for sensorless switched reluctance motor drives," in IEEE Transactions on Power Electronics, vol. 15, no. 2, pp. 319-334, March 2000.

[13] S. Rehman and D. G. Taylor, "Issues in position estimation of sr motors," in IEEE Power Electron. Spec. Conf. Rec., 1996, pp. 337-343.

[14] B. Fahimi, G. Suresh, J. Mahdavi, and M. Ehsani, “A New Approach to Model Switched Reluctance Motor Drive Application to Dynamic Performance Prediction, Control and Design," The 29th IEEE Power Electronics Specialists Conference, Vol. 2, May 17-22, 1998, pp. $2097-$ 2102 